\title{
ÉTUDE DU TRANSIT ÉPIDIDYMAIRE DES SPERMATOZOÏDES DE TAUREAU MARQUÉS A L'AIDE DU ${ }^{32} P$
}

\author{
Marie-Claire ORGEBIN \\ Station de Recherches de Physiologie animale, \\ Centre national de Recherches zootechniques, Jouy-en-Josas.
}

\begin{abstract}
SOMMAIRE
La vitesse de passage des spermatozoïdes dans l'épididyme du Taureau a été déterminée en injectant par voie intra-veineuse $\mathrm{du}^{32} \mathrm{P}$.

Les spermatozoïdes à acide désoxyribonucléique (ADN) marqué apparaissent au plus tôt $4 \mathrm{r}$ jours après l'injection, dans la partie proximale de la tête de l'épididyme, et seulement 49 à 52 jours après l'injection, dans l'éjaculat.

La différence entre ces deux valeurs indique que les spermatozoïdes mettent 8 à I I jours pour traverser l'épididyme : valeur très inférieure à celle du Bélier.
\end{abstract}

Ces dernières années DAwson, I958, KoEFOED-Johnsen, I958, ORGEBINCourot-OrTaVANt, ont montré que, chez le Taureau, 48 à 52 jours après l'injection de ${ }^{32} \mathrm{P}$, des spermatozoïdes à acide désoxyribonucléique $(\mathrm{ADN})$ marqué apparaissent dans l'éjaculat.

Pour estimer la part qui revient dans ce délai au transit épididymaire, KoEFOED-JOHNSEN (I959) pense qu'il est raisonnable d'appliquer au Taureau les résultats obtenus chez le Bélier (OrTavaxts, I954) et chez le Lapin (KoEFoED-Johnsen, I959). Chez ces espèces, les spermatozoïdes à ADN marqué arrivent dans la tête de l'épididyme 30 à $3 \mathrm{I}$ jours après 1 'injection de ${ }^{32} \mathrm{P}$, il en résulterait_donc une durée de transit épididymaire de I7 à I9 jours.

DAwson, expérimentant sur un Taureau, calcule que la transformation des spermatocytes primaires en spermatozoïdes nécessite 39 jours. La durée de séjour des spermatozoïdes dans l'épididyme serait alors de Io à I3 jours.

En présence de ces conclusions différentes, nous avons voulu préciser cette durée du transit épididymaire par une mesure directe du déplacement des spermatozoïdes radioactifs dans l'épididyme.

\section{MATÉRIFL ET MÉTHODES}

Nous avons injecté à 22 taureaux adultes par voie intraveineuse de 0,05 à $0,1 \mathrm{mc} / \mathrm{kg}$ de poids vif de ${ }^{32} \mathrm{P}$ sous forme de $\mathrm{PO}_{4} \mathrm{HNa}_{2}$.

Annales de Biologie animale. - 196x. 


\section{A. - Récolte des spermatozoÏdes ÉJaculés :}

Les spermatozoïdes sont recueillis au moyen d'un vagin artificiel. Motilité, concentration et proportion de spermatozoïdes vivants sont déterminées pour chaque éjaculat.

Les spermatozoïdes sont séparés du plasma séminal par centrifugation pendant $20 \mathrm{mn}$ à $0^{\circ} \mathrm{C}$, puis lavés 5 fois toujours à $0^{\circ} \mathrm{C}$ dans une solution physiologique de Krebs-Henseleit-Ringer (ManN, I954).

\section{B. - RÉCOLTE DES SPERMATOZOÏDES ÉPIDIDYMAIRES :}

Les animaux sont castrés bilatéralement; l'épididyme est prélevé et découpé en 5 fractions, la tête et la queue étant seules séparées en une partie proximale et une partie distale. Après enlèvement de l'albuginée chacune de ces $\mathrm{f}$ ractions est dilacérée finement à l'aide de ciseaux dans une solution saline hypotonique (I00 $\mathrm{cm}^{3}$ de Krebs-Ri nger $+400 \mathrm{~cm}^{3}$ d'eau bidistillée) pour assurer une meilleure élimination des hématies dans la suite des manipulations. Après macération pendant $30 \mathrm{mn}$ à $0^{\circ} \mathrm{C}$, les spermatozoïdes de chaque fraction sont récupérés par filtration sur 6 couches de gaze. Les spermatozoïdes ainsi recueillis sont lavés avec la solution saline hypotonique et centrifugés 3 fois à o ${ }^{\circ} \mathrm{C}$, pendant Io $\mathrm{mn}$ à 5 ooo tours/mn. Le culot de spermatozoïdes ainsi obtenu est débarrassé à peu près complètement des hématies et de toute cellule étrangère.

\section{C. - Analyse chimique des spermatozoïdes :}

Les différentes fractions phosphorées des spermatozoïdes sont séparées par la technique de Schmidt et Thannhauser (1945), légèrement modifiée (OrTavant, 1949). La suspension lavée des spermatozoïdes obtenus soit par collecte, soit par castration est homogénéisée au moyen d'un POTTERELVEHJEM dans to volumes d'acide trichloracétique (ATC) Io p. I00 à $0^{\circ} \mathrm{C}$. La suspension est laissée $30 \mathrm{mn}$ à cette même température puis centrifugée à froid pendant Io $\mathrm{mn}$ à 5000 tours/mn. Le résidu est ainsi extrait 2 fois et lavé avec de l'eau bidistillée à o ${ }^{\circ} \mathrm{C}$.

Le résidu est ensuite mis en suspension dans io volumes d'un mélange chloroforme-méthanol (2-I) et laissé une heure à $18^{\circ} \mathrm{C}$. Après centrifugation, le résidu est extrait 2 fois, lavé dans 5 volumes d'éther et séché sous ventilateur à la température ambiante. $100 \mathrm{mg}$ de résidu sec ainsi délipidé, sont alors hydrolysés avec ro $\mathrm{cm}^{3}$ d'une solution de $\mathrm{NaOH}, \mathrm{N}$ à $37^{\circ} \mathrm{C}$ pendant 20 heures ; une bonne dissolution du résidu est assurée au moyen d'une agitation temporaire. Après centrifugation à $0^{\circ} \mathrm{C}$, l'ADN $^{\prime}$ est précipité à froid avec 0,2 volume de $\mathrm{C}_{\mathrm{II}} \mathrm{I}, 6 \mathrm{~N}$ et I volume d'ATC $5 \mathrm{p}$. Ioo. Le précipité est lavé 3 fois avec $5 \mathrm{~cm}^{3}$ d'ATC 5 p. Ioo, puis extrait pendant $15 \mathrm{mn}$ par $5 \mathrm{~cm}^{3}$ d'ATC p. Ioo à $90-95^{\circ} \mathrm{C}$.

Après centrifugation on obtient une fraction contenant l'acide désoxyribonucléique dont le phosphore est dosé selon la technique d'ALLEN (1940). La radioactivité spécifique est déterminée sur une partie aliquote de la fraction extraite, mise dans une coupelle de verre, séchée sous une lampe infrarouge et portée sous un compteur Geiger-Müller. Les résultats sont toujours rapportés au jour de l'injection.

\section{RÉSULTATS}

En castrant Io Taureaux à des temps différents après l'injection de ${ }^{32} \mathrm{P}(36,38$, $40,4 I, 42,43$ jours) nous avons observé que jusqu'au $40^{\circ}$ jour après l'injection, il n'y avait aucun spermatozoïde à $A D N$ radioactif dans l'épididyme. Les spermatozoïdes marqués apparaissent d'une façon constante le $4 \mathrm{I}^{\mathrm{e}}$ jour après l'injection dans la partie proximale de la tête de l'épididyme (fig. I).

Pour détecter leur arrivée dans la partie distale de la queue de l'épididyme nous avons simplement suivi les variations de la radioactivité des spermatozoïdes de l'éjaculat puisque ceux-ci proviennent des ampoules du canal déférent et de la partie distale de la quetue de l'épididyme.

Nous avons effectué ces mesures sur I2 animaux. Les quantités de spermatozoïdes récoltés et les fréquences de collectes étaient différentes pour chaque expérience : 2 Taureaux n'ont pas été collectés durant les 49 jours qui suivent l'injection de 


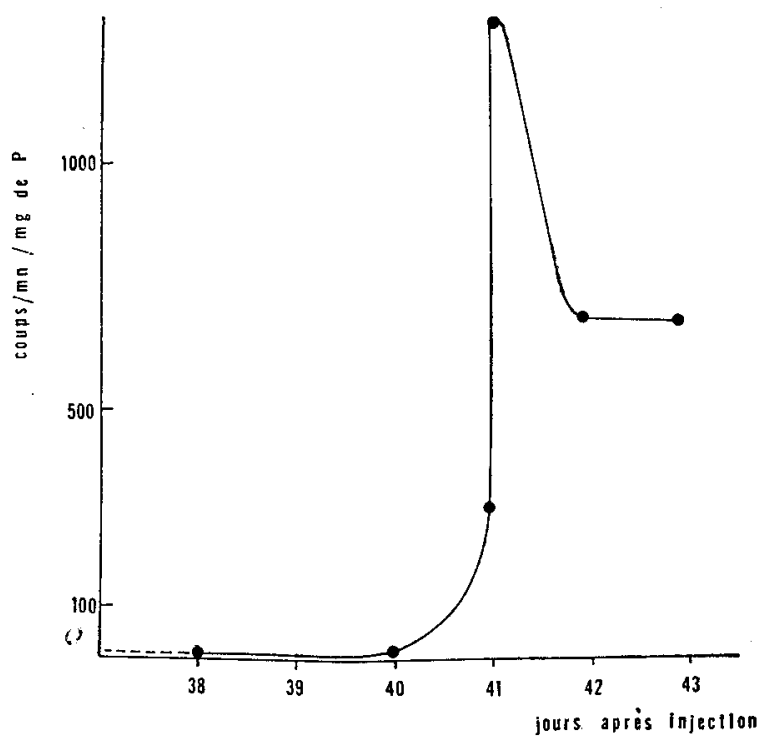

FIG. I - Variation de la radioactinité spécifique de l'ADN des spermatozoiddes de la partie proximale de la lête de l'épidyme de Taureaux castrés à des temps différents après l'injection de ${ }^{32} P$.

Cette radioactivité augmente brusquement le $4 \mathrm{I}^{\mathrm{\theta}}$ jour après l'injection indiquant l'arrivée des spermatozoïdes marqués dans cette partie de l'épididyme.

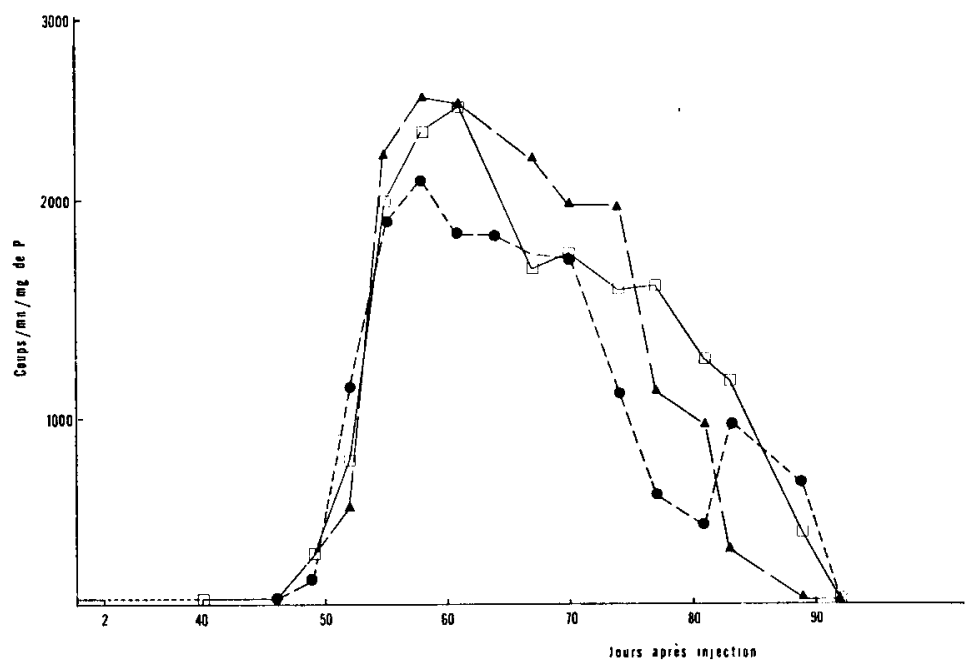

FIG. 2 -Variation de la radioaclivité spécifique de l'ADN des spermalozö̈des dans l'éjaculat de Taureaux ayant recu des doses de ${ }^{32} \mathrm{P}$ de 0,05 à $0,1 \mathrm{mc} / \mathrm{kg}$ de poids vif.

L'apparition de ces spermatozoïdes radioactifs a lieu $49^{-5} 5^{2}$ jours après l'injection.

${ }^{32} \mathrm{P}, 8$ ont été collectés à des fréquences variables, 2 enfin ont été soumis à des épuisements complets. Malgré l'obtention de $\overline{0}$ milliards de spermatozoïdes par jour, dans le cas des collectes les plus fréquentes, nous n'avons jamais trouvé de spermatozoïdes à $A D N$ marqué dans 1'éjaculat avant le $48^{\mathrm{e}}$ jour, l'apparition la plus tardive ayant, lieu $1 \mathrm{e} 52^{\mathrm{e}}$ jour (fig. 2). 
On peut donc déduire de ces 2 séries d'expériences que le temps mis par les spermatozoïdes pour traverser l'épididyme est au minimum de 8 jours et au maximum de II jours.

Ainsi la durée de séjour des spermatozoïdes dans l'épididyme de Taureau est plus courte que celle trouvée chez le Bélier par Or'TAVANT (I955) (I3-2I jours).

On peut rapprocher ceci du fait que les réserves spermatiques épididymaires sont chez cet animal beaucoup plus importantes que chez le Taureau (OrTAvant I956).

Il existe une autre différence assez remarquable: chez le Bélier les spermatozoïdes à $A D N$ marqué arrivent dans la tête de l'épididyme 30-3x jours après 1'injection de ${ }^{32} \mathrm{P}$ et chez le Taureau $4 \mathrm{I}$ jours seulement après l'injection. Si les stades d'incorporation du ${ }^{32} \mathrm{P}$ dans 1'ADN sont les mêmes chez ces deux animaux, on peut supposer que le déroulement de la spermatogenèse nécessite plus de temps chez le Taureau alors que la durée de maturation épididymaire est plus courte.

\section{Reçu en mars 1960.}

\section{SUMMARY}

\section{A STUDY OF EPIDIDYMAL TRANSPORT IN BULL USING ${ }^{32} \mathrm{p}$}

In order to determine the rate of passage of spermatozoa in the epididymis of the bull. 22 adult bulls were injected intravenously with 0,05 to $0,1 \mathrm{mc}$ of ${ }^{32} \mathrm{P}$ (in the form of $\mathrm{PO}_{4}\left[\mathrm{INa}_{2}\right.$ ) per $\mathrm{kg}$ body weight.

The different phosphorus compounds of sperm were extracted following the Schmidt and THanNHAUSER method and their specific radioactivity was determined.

Io bulls were castrated at various times after the injection of ${ }^{32} \mathrm{P}$. Spermatozoa with radioactive DNA appear in the proximal part of the head of the epididymis, at the earliest, 4I days after the injection (fig. I).

Collections of sperm were made from 12 bulls. The first spermatozoa with labelled DNA began to appear in the ejaculate 49 to 52 days after the injection (fig. 2 ).

From these experiments it can be concluded that it takes 8 to I I days for spermatozoa to pass through the epididymal canal.

Thse results indicate that, in the bull, spermatogenesis is longer whereas the rate of passage through epididymis is shorter than in the ram.

\section{RÉFÉRENCES BIBLIOGRAPHIQUES}

AlLEN R. J. L., I940. 'The estimation of phosphorus. Bioch. J., 34, 858-865.

Dawson R. 11. C., 1958. Labclling of Bull semen with Phosphorus-32 in vivo. Nature, 181, ror4-Ior 5.

KOEFOED-JoHnseN H. H., I958. Investigations on sperm formation. Roy. Vet. Agric. Coll. Sterility Res. Inst. Ann. Rep., $18-33$.

KOEFond-Jomsisen H. H., 1959. Influence of Jijaculation Frequency on the Time required for Sperm Formation and Epididymal Passage on the Bull. Nature, 185, 49-5o.

MANN T., TO54. The biochemistry of scmen, [. 77 , Melluten an Co, LTD J.ondon.

Orgeisix M. C., Colrot M., Ortavant R., cité dans Cole H. Il. et Cuples P. T., i959. Reproduction in Domestic Animals, 11, 4I-43, Academic Press, New-York and London.

Ortavant R., ig54. Contribution à l'étude de la durée du processus spematogénétique du Bélier à l'aide du 3:P.C. R. Soc. Biol., 148, 804-806.

ORtavant R., I955. Etude sur la spermatogenèse des animaux domestiques à l'aide du phosphore- $3^{2}$. Conférence internationale sur l'utilisation de l'énergie atomique à des fins pacifiques, 12, 243-245.

ORTavant R., 1956. Spermatogenèse ct réserves spermatiques chez le Taureau et le liélier. IIIrd́ Intern. Cong. Anim. Reprod. Cambridge, p. 44.

Ortavant R., 1959. Déroulement et durée du cycle spermatogénétique chez le Bélier (premicre partie), Ann. Zoolech., 8, $18,3-249$.

Scimint G., Thanninausir S. J., 1945. A method for the detemination of desoxyribonucleic acid, ribonucleic acid and phosphoproteins in animal tissues. J. Biol. Chem., 161, 83-89. 